usually not on the national recall system and who appear to be at increased risk of cervical abnormalities due to the presence of sexually transmitted infections such as genital warts which are known to be linked with cervical dysplasia.

A large age matched, controlled, prospective study is, however, needed to validate the impression that women attending STD clinics are a "high risk" group for cervical cytological abnormalities and that the abnormalities detected are clinically significant to warrant a policy of opportunistic screening.

TYOTI DHAR $M$ BRADLEY AB ALAWETTAGAMA Department of Genitourinary Medicine, Royal Liverpool University Hospital

Address correspondence to Dr Dhar

1 NHS National Coordinating Network Workshop. The role of Genito-Urinary Medicine cytology and colposcopy in cervical screening. Final version, December 1991.

2 Coleman DV. Efficient use of cervical screening. Lance 1987;ii:510-1.

3 DHSS. Health Services Management: Cervical cancer screening. (Health Circular HC [88] 1). DHSS: Heywood, Lancs, 1988.

4 Beardow R, Oerton J, Victor CR. Evaluation of the cervical cancer screening programme in an inner city health district. BMF 1989;299:98-100

5 Holland W, Stewart S. Screening in health care. London: Nuffield Provincial Hospitals Trust, 1990.

6 Amery J, Beardow R, Oerton J, Christina V. The efficacy of a national Family Health Services Authority based cervical cytology system. Health Trends 1992;24:119-22

Accepted for publication 9 July 1993

\section{Establishing a satellite genitourinary medicine clinic}

In urban areas a single genitourinary medicine (GUM) clinic may serve a large population. In rural areas with a low population density, smaller local clinics may be required because otherwise patients may be unable to attend an appropriate clinic. In April 1992 we set up a satellite clinic at a health centre in the centre of the English Lake District as an adjunct to a larger clinic located at a hospital 15 miles away. Travel between these locations by public transport is very difficult, and many young people do not have a car. The locality includes many hotels and restaurants and an establishment for higher education. The local general practitioners (GPs) supported the initiative.

Two good clinical rooms and a suitable patient waiting area were available at the health centre for a clinic session of an hour's duration once every two weeks. The clinic was discretely signposted. The clinic times were advertised to local GPs and at selected sites such as the educational establishment and at staff residences in hotels. The GUM consultant and health adviser from the hospital were assisted by the surgery practice nurse and one of the local GPs who provided much helpful local knowledge. No receptionist was necessary. Case notes were stored at the hospital clinic and current notes were transported to the satellite clinic.
We aimed to keep tests and treatment simple because patients requiring complicated tests and treatments could be referred easily to the hospital clinic. Wet microscopy of vaginal preparations was performed using a portable microscope taken to the clinic but we managed without gram-staining slides. Identification of gonorrhoea, chlamydia and herpes virus was undertaken using suitable transport media. Serological tests for syphilis, hepatitis B and HIV were available. All specimens were taken to the hospital laboratory immediately after the clinic, and results were directed to the hospital clinic to ensure that confidential reports did not get lost in the surgery's mail. A small stock of standard medicines was taken to the the clinic. Genital warts were treated with applications of TCAA and podophyllum.

In the first year, 28 clinics of one hour were held and there were 123 attendances, including 63 new patients. No cases of syphilis, gonorrhoea or HIV infection were detected. There were six cases of chlamydia and eight patients were treated for nonspecific urethritis or epidemiologically for non-specific urethritis or chlamydia. There were 23 cases of genital warts, three cases of primary genital herpes, 12 of candida and seven of bacterial vaginosis. After pre-test discussion with the health adviser 50 patients were tested for HIV. Almost all patients belonged to the target population of young sexually active people, and several commented on the convenience of attending a local clinic. One patient attending regularly for wart treatment pointed out that she could tell her employer that she was popping out to the doctors for ten minutes, whereas a trip to the hospital clinic would have required an explanation for a half-day absence. No problems were encountered in providing a GUM service within a GP surgery setting. Attendance for follow-up visits was good and contact tracing success was excellent. Towards the end of the year a further satellite clinic was established at another location and we now provide a satellite clinic session each week, alternating between the two sites.

In conclusion, the satellite clinic provided an effective low-cost addition to the main hospital clinic in controlling sexually transmitted diseases. The success depended upon favourable features of the locality, including the support of staff at the only major health centre in the area, and the involvement of an experienced GUM doctor and health adviser who were able to maintain standards of practice similar to those at a hospital department of GUM. Without these features, a GUM clinic in a GP surgery might not exert a beneficial effect on public health.

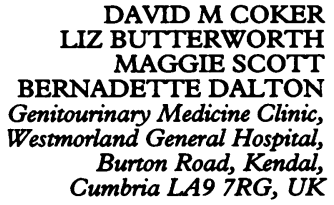

Address correspondence to Dr Coker Accepted for publication 18 June 1993 